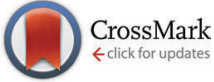

Cite this: Phys. Chem. Chem. Phys., 2015, 17, 25956

Received 20th April 2015, Accepted 7th July 2015

DOI: $10.1039 / c 5 c p 02304 a$

www.rsc.org/pccp

\section{Nonlinear effects in infrared action spectroscopy of silicon and vanadium oxide clusters: experiment and kinetic modeling $\dagger$}

\author{
Florent Calvo, ${ }^{a}$ Yejun Li, ${ }^{b}$ Denis M. Kiawi, ${ }^{\text {cd }}$ Joost M. Bakker, ${ }^{c}$ Pascal Parneix ${ }^{e}$ and \\ Ewald Janssens ${ }^{b}$
}

\begin{abstract}
For structural assignment of gas phase compounds, infrared action spectra are usually compared to computed linear absorption spectra. However, action spectroscopy is highly nonlinear owing to the necessary transfer of the excitation energy and its subsequent redistribution leading to statistical ionization or dissociation. Here, we examine by joint experiment and dedicated modeling how such nonlinear effects affect the spectroscopic features in the case of selected inorganic clusters. Vibrational spectra of neutral silicon clusters are recorded by tunable IR-UV two-color ionization while IR spectra for cationic vanadium oxide clusters are obtained by IR multiphoton absorption followed by dissociation of the bare cluster or of its complex with Xe. Our kinetic modeling accounts for vibrational anharmonicities, for the laser interaction through photon absorption and stimulated emission rates, as well as for the relevant ionization or dissociation rates, all based on input parameters from quantum chemical calculations. Comparison of the measured and calculated spectra indicates an overall agreement as far as trends are concerned, except for the photodissociation of the $\mathrm{V}_{3} \mathrm{O}_{7}^{+}-\mathrm{Xe}$ messenger complex, for which anharmonicities are too large and poorly captured by the perturbative anharmonic model. In all systems studied, nonlinear effects are essentially manifested by variations in the intensities as well as spectral broadenings. Differences in some band positions originate from inaccuracies of the quantum chemical data rather than specific nonlinear effects. The simulations further yield information on the average number of photons absorbed, which is otherwise unaccessible information: several to several tens of photons need to be absorbed to observe a band through dissociation, while three to five photons can be sufficient for detection of a band via IR-UV ionization.
\end{abstract}

\section{Introduction}

The electronic and structural properties of molecules and clusters produced in the gas phase can be conveniently addressed by spectroscopic methods probing different wavelength ranges.

${ }^{a}$ Laboratoire Interdisciplinaire de Physique, Rue de La Piscine, Campus Saint Martin d'Hères, 38000 Grenoble, France. E-mail: florent.calvo@ujf-grenoble.fr; Fax: +33 4766354 95; Tel: +33476514592

${ }^{b}$ Laboratory of Solid State Physics and Magnetism, KU Leuven, Celestijnenlaan 200 D, B-3001 Leuven, Belgium

${ }^{c}$ Radboud University, Institute for Molecules and Materials, FELIX Laboratory, Toernooiveld $7 c, 6525$ ED Nijmegen, The Netherlands

${ }^{d}$ Anton Pannekoek Institute, University of Amsterdam, Science Park 904, 1098 XH Amsterdam, The Netherlands

${ }^{e}$ Institut des Sciences Moléculaires d'Orsay, UMR CNRS 8214, Université Paris Sud 11, Bât. 210, F91405 Orsay Cedex, France

$\dagger$ Electronic supplementary information (ESI) available: Detailed quantum chemical data for all clusters studied, harmonic IR absorption spectra of bare and xenon-tagged $\mathrm{V}_{3} \mathrm{O}_{7}^{+}$, and a comparison between measured and modelled spectra for $\mathrm{Si}_{10}$. See DOI: $10.1039 / \mathrm{c} 5 \mathrm{cp} 02304 \mathrm{a}$
The low densities of particles in the gas phase prevent the use of direct absorption spectroscopy. This limitation can be circumvented by action techniques using mass spectrometry to monitor the outcome of laser excitation by a change in the mass or the charge state of the studied species. ${ }^{1,2}$ Such a change conveys the destabilization of the species upon absorbing sufficient amounts of energy at a given excitation frequency. With the parallel development of intense and stable sources based on free-electron lasers (FELs) ${ }^{3,4}$ or synchrotron light, action spectroscopy experiments have seen major progress in the recent years. ${ }^{1,2,5-9}$

For complex molecules, structural assignment by action spectroscopy methods requires comparison with computed spectra. In recent decades, advances in quantum chemistry methods and computer hardware have established density functional theory (DFT) as the framework of choice to compute IR and optical spectra. ${ }^{10-14}$ This approach has well known limitations such as the poor description of long-range dispersion forces, strong correlation regimes or, in the case of time-dependent 
DFT for optical spectra, charge transfer excitations. Despite such restrictions, the DFT approach remains quite robust, relatively free of parameters, and computationally more efficient than most wavefunction-based methods.

The increasing success of action spectroscopy experiments coupled with DFT calculations relies on the accuracy of the quantum chemical method, but also on the validity of several underlying approximations inherent to those calculations. The frequencies predicted by this approach are often off by a significant amount that depends on the system and the details of the calculation. The introduction of scaling factors to address these discrepancies ${ }^{15-19}$ is a cheap empirical way of addressing their actual causes, namely anharmonicities of the potential energy and dipole moment surfaces and the related thermal or quantum delocalization effects to which they are sensitive. This should in principle not be necessary, as the current computing power allows for the determination of anharmonicities using vibrational perturbation theory (VPT2), ${ }^{20}$ vibrational selfconsistent field (VSCF) ${ }^{21}$ configuration interaction $(\mathrm{VCI})^{22}$ or, in larger systems, by classical $^{23}$ or quantum ${ }^{24,25}$ molecular dynamics possibly built on explicit descriptions of the electronic structure. $^{6,26}$

Besides intrinsic anharmonic effects on the absorption frequency, another essential limitation of conventional computational methods is the systematic evaluation of linear absorption intensities for comparison to experimental spectra. Action spectroscopy is intrinsically a nonlinear technique with additional mechanisms separating the photon absorption from the detected signal through energy redistribution processes and, in the case of dissociation, subsequent energy localization in the decaying modes. Nonlinear dependencies of spectral features (band positions, widths and intensities) on the laser characteristics, have been previously duly noted ${ }^{27-29}$ and analysed in the case of IR multiphoton dissociation (IR-MPD) and IR resonanceenhanced multiphoton ionization (IR-REMPI) spectroscopies for hydrocarbon and fullerene molecules, ${ }^{30-32}$ and for the predissociation of small compounds. ${ }^{33}$ Except these investigations, we are not aware of earlier attempts to model the dependence of IR spectra of gas phase species on the experimental conditions.

Recently, we made a description of nonlinear effects in vibrational action spectroscopy from a molecular perspective, either directly at the atomistic level through explicit time-dependent molecular dynamics ${ }^{34}$ or from a discrete quantum mechanical description of energy levels. ${ }^{35}$ Although these approaches rely on different assumptions and make use of a complementary treatment for the nuclear dynamics and the interaction with the laser field, they consistently predict action spectra that vary with the excitation pulse and systematically differ from the linear absorption spectra even after anharmonicities are accounted for. These predictions are consistent with experimental measurements. ${ }^{35,36}$ However, no systematic analysis of nonlinear effects has been reported yet. Understanding their importance would improve vibrational action spectroscopy and promote it as a powerful analytical technique for structural characterization.

In this paper we report a combined experimental and computational study of such issues. As model systems inorganic clusters were chosen whose structures have already been characterized by IR spectroscopy, namely $\mathrm{Si}_{6}, \mathrm{Si}_{7}, \mathrm{Si}_{10}, \mathrm{~V}_{4} \mathrm{O}_{10}{ }^{+}$and $\mathrm{V}_{3} \mathrm{O}_{7}{ }^{+}$. IR-UV two-color ionization and IR-MPD spectra were measured for neutral silicon and cationic vanadium oxide clusters, respectively. Photoionization and dissociation propensities were modeled using the same approach as in ref. 35, and adapted to the present problems. Our results generally indicate that nonlinear effects on the vibrational spectra are significant and manifested mostly as changes in intensity and, to a lesser extent, to some broadening as the photon exposure, macropulse duration or laser bandwidth are varied. For the present systems, no significant spectral shifts are found. Although modeled and experimental spectra generally agree, some discrepancies are found for absolute intensities near the saturation regime, and for xenon-tagged vanadium oxide clusters. Those discrepancies are likely due to inaccuracies in the underlying quantum chemical data, notably caused by a poorly converging anharmonic expansion in presence of the xenon messenger atom. The article is organized as follows. In the next section the experimental and computational modeling tools are described. Subsequently, results on silicon and vanadium oxide clusters are presented and discussed in Sections 3 and 4, respectively. Concluding remarks are given in Section 5 .

\section{Methodology}

\subsection{Experimental}

The far-IR spectroscopy experiments are performed at the Free Electron Lasers for infrared eXperiments (FELIX) laboratory. ${ }^{3}$ The clusters are produced in a molecular beam setup, which is connected to a beam line of FELIX. ${ }^{37}$ The instrument contains a new laser vaporization cluster source and a time-of-flight mass spectrometer. The entire experimental setup is depicted in Fig. 1. The 2nd harmonic of a pulsed $(10 \mathrm{~Hz}) \mathrm{Nd}$ :YAG laser ablates a solid target rod of silicon (Goodfellow, 99.999\%) or vanadium (Sigma Aldrich, 99.7\% purity). A short pulse of helium subsequently cools the formed plasma and promotes cluster formation through carrier gas mediated collisions in the clustering channel. For the vanadium oxide clusters $0.3 \%$ of $\mathrm{O}_{2}$ gas is added to the He carrier gas, yielding oxygen saturated cluster growth conditions. In addition the carrier gas contains $1.0 \%$ of Xe gas, which is needed for the formation of the cluster-rare gas messenger complexes. Isotopically enriched ${ }^{129} \mathrm{Xe}$ is used to reduce the number of isotopomers present in the mass spectra. The clustering channel is extended by a $45 \mathrm{~mm}$ long copper flow tube, whose temperature is kept

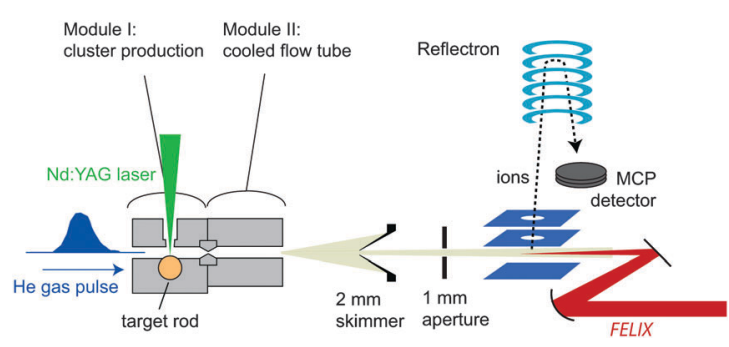

Fig. 1 Schematic of the experimental setup. 
constant at $T_{0}=110 \mathrm{~K}$ (for silicon clusters) and $170 \mathrm{~K}$ (for vanadium oxide clusters) by a combination of liquid nitrogen cooling and resistive heating. At the end of the channel the gas pulse is expanded into vacuum. The resulting molecular beam is shaped first by a $2 \mathrm{~mm}$ skimmer and, after traveling through a differential vacuum chamber, by a second aperture (of $1 \mathrm{~mm}$ diameter), which ensures a good spatial overlap between the molecular beam and the IR laser beam. About $50 \mu$ s after interaction with the laser, cationic clusters are pulse-extracted into a reflectron time-of-flight mass spectrometer. For the study of neutral silicon clusters the second aperture is placed at a potential to deflect charged particles out of the molecular beam. The neutral clusters are then post-ionized by an unfocused beam of an $\mathrm{F}_{2}$ gas laser (7.9 eV per photon), right before extraction into the mass spectrometer.

The experiment runs at twice the FELIX repetition rate ( $5 \mathrm{~Hz}$ ), thus recording mass spectra with and without IR excitation. The latter serve as reference mass spectra that account for cluster production fluctuations. FELIX produces IR radiation in the $70-3600 \mathrm{~cm}^{-1}$ spectral range, although for the current experiments only selected spectral ranges $\left(390-500 \mathrm{~cm}^{-1}\right.$ for $\mathrm{Si}_{n}$ and 1000-1090 $\mathrm{cm}^{-1}$ for $\mathrm{V}_{n} \mathrm{O}_{m}{ }^{+}$) are scanned, corresponding to the most intense absorption modes as identified before. ${ }^{11,38-40}$ Multiple scans of the same spectral range were carried out under different experimental conditions (photon exposure, macropulse duration, laser bandwidth). To the extent possible only one parameter was changed at a time. The FELIX laser pulses are produced in a pulse train, the so-called macropulse, with a typical duration of 3-10 $\mu$ s (after subtraction of $2 \mu \mathrm{s}$ for build-up of the cavity power), consisting of ps-long micropulses at a $1 \mathrm{~ns}$ separation. For the experiments described here typical macropulse energies used are about 15-50 mJ (before attenuation), resulting in a typical photon exposure (or integrated photon fluence) of $2-6 \times 10^{24}$ photons per $\mathrm{m}^{2}$. To vary the photon exposure fixed-value attenuators are used. The radiation is near transform-limited and the spectral width can be adjusted between typically $0.1 \%$ and $1 \%$ RMS of the central frequency.

The duration of the macropulse and the bandwidth are known within $10 \%$ and $20 \%$ accuracy, respectively. The photon exposure is known within a factor of two. This large uncertainty is mainly due to the uncertainty of the laser beam radius at the point where the light interacts with the clusters. It concerns a systematic uncertainty when comparing photon exposures from different measurements. The relative uncertainty (laser power fluctuations, inaccuracy in the power measurement) is of the order of only $15 \%$. Besides this uncertainty, the laser beam has a nearly Gaussian spatial profile. This implies that the photon exposure is stronger near the center of the beam than at the edges. For a perfect overlap of the molecular beam $(0.5 \mathrm{~mm}$ radius) and at the focus of the laser beam with a waist of $0.5 \mathrm{~mm}$, a photon exposure in the center of $10^{24} \mathrm{~m}^{-2}$ corresponds to one of $0.14 \times 10^{24} \mathrm{~m}^{-2}$ at the edges.

\subsection{Modeling}

In order to evaluate the influence of excitation conditions on the vibrational spectra, nonlinear effects associated with the various physical mechanisms of photon absorption, spontaneous and stimulated photon emission, and the relevant dissociation or ionization process must be taken into account. We follow here a kinetic Monte Carlo (kMC) approach that treats those processes based on an quadratic expansion in energy levels, also known as Dunham expansion. The method has been explained in details in a recent publication, ${ }^{35}$ so only its main features are given here.

Briefly, the vibrational level structure is expanded at second order within perturbation theory, ${ }^{20}$

$$
E(\mathbf{N})=E_{0}+\sum_{i} \hbar \omega_{i}\left(n_{i}+\frac{1}{2}\right)+\sum_{i \leq j} \chi_{i j}\left(n_{i}+\frac{1}{2}\right)\left(n_{j}+\frac{1}{2}\right)
$$

where $E_{0}$ is a constant, $\mathbf{N}=\left\{n_{i}\right\}$ a series of $\kappa$ vibrational quantum numbers associated with harmonic frequencies $\omega_{i}$ and anharmonic coupling constants $\chi_{i j}$, respectively. All those ingredients are provided by quantum chemical calculations at the DFT level. The system is described statistically by its vibrational population $\mathbf{N}$ or equivalently by its internal energy $E$; the two quantities are connected through the vibrational density of states $\Omega(E)$.

In the kinetic modeling, the system is evolved as a function of time by enumerating all possible events such as photon absorption and stimulated emission (during exposure to the laser pulse), spontaneous emission and possible statistical dissociation. All radiative events are characterized in terms of the energy difference $\hbar \omega$ they are associated with, and the corresponding rates are evaluated using the harmonic approximation for the oscillator strengths. Those quantities require the Einstein coefficients for transitions from (or to) the ground vibrational state, which are provided by the quantum chemical calculations through the IR absorption intensities. Rates for photoionization of neutral $\mathrm{Si}_{n}$ clusters and for dissociation of cationic $\mathrm{V}_{n} \mathrm{O}_{m}{ }^{+}$clusters are discussed in Sections 3 and 4 , respectively.

Instead of working directly in the discrete space of levels $\mathbf{N}$, all rates are evaluated as a continuous function of the internal energy $E$. In between two consecutive events, the system is treated in the microcanonical ensemble. All relevant statistical rates are obtained by multicanonical Monte Carlo simulations on the space of vibrational levels where the normalizing density of states $\Omega$, required to achieve uniform sampling, is predetermined using the Wang-Landau method. ${ }^{41,42}$ The microcanonical density of states also provides access to the level distributions at thermal equilibrium and to the IR absorption spectrum at finite temperature, which thus includes finite temperature anharmonic effects, yet still in the linear regime. ${ }^{43}$ It will be shown below that the absorption spectra, at the rather low temperatures at which the clusters are generated, are rather similar to the static spectra at $0 \mathrm{~K}$. However the action spectra are significantly different, thus reflecting direct heating effects associated with multiphoton absorption.

A key assumption of the kMC procedure is that it is a Markov process in which the system has no memory of the previous 
state and a complete intramolecular vibrational redistribution (IVR) of energy occurs between two successive events. At time $t=0$ the energy is randomly drawn from a thermal population at fixed experimental temperature $T_{0}$. The rates $k(\omega, E)$ of all events are calculated and one such event is randomly drawn proportionally to $k$. The event is performed with the time modified accordingly, and the new list of events and their associated rates are evaluated for this microstate. The Monte Carlo trajectory is then propagated until the time reaches a maximum delay since excitation. In the case of IR-UV two-color ionization of neutral silicon clusters, this delay is the time between the IR and UV lasers pulses, and in the case of dissociation of vanadium oxide clusters it is the time until extraction. Finally, the probabilities of dissociating or ionizing the cluster after fixed observation times $t_{\text {obs }}$ are estimated from $10^{5}$ independent kMC trajectories, and the resulting simulated action spectrum is convoluted by a Gaussian with full width at half maximum (fwhm) of $4\left(B k_{\mathrm{B}} T_{0}\right)^{1 / 2}$ in order to account for rotational broadening, ${ }^{44} B$ being the average rotational constant of the molecule and $k_{\mathrm{B}}$ the Boltzmann constant.

The laser characteristics enter the modeling mainly in the pulse duration $\Delta t$ and in the absorption and stimulated emission rates, which are both proportional to the photon fluence rate $\Phi(\nu)$ with a Gaussian-shape spectrum centered at $\nu_{\mathrm{L}}$ and with a spectral width $\Delta \nu_{\mathrm{L}}$ assumed to be proportional to $\nu_{\mathrm{L}}$. Two assumptions in our modeling should be emphasized, namely (i) that the micropulse structure of the laser, which is characteristic for a free-electron laser, is neglected and only a single macropulse is simulated; and (ii) a uniform exposure is assumed to be experienced by all clusters instead of a more realistic Gaussian spatial laser beam profile. The neglect of the micropulse structure is dictated by practical convenience, its time resolution being incompatible with the kinetic Monte Carlo approach. We show below that for all systems studied, the total number of photons absorbed remains limited. The chance that more than one photon is absorbed per individual micropulse is thus highly unlikely. The uniform laser beam profile employed in the simulations was chosen also in order to keep the computations tractable, as much larger statistics should be needed to cover the spatial dependence of the photon fluence.

In contrast to the vast majority of static DFT calculations in the harmonic approximation, we have not applied any scaling factor to match the calculated lines to experimental bands, nor did we empirically modify the calculated action spectra for better reproducing the measured spectra. Differences in peak positions thus reflect the inherent errors of the quantum chemical methodology.

\section{IR-UV two-color ionization of silicon clusters}

Owing to their importance in materials science and potential applications in nanoelectronics, silicon clusters have received a sustained attention from experimentalists and theoreticians. ${ }^{45}$
Their geometric and electronic structures have notably been characterized using photoelectron spectroscopy, ${ }^{46-48}$ ion mobility spectrometry, ${ }^{49}$ and more recently vibrational spectroscopy. IR-MPD spectroscopy of cluster-rare gas complexes was used both for cationic and neutral silicon clusters. ${ }^{39,50}$ Neutral $\mathrm{Si}_{6}$, $\mathrm{Si}_{7}, \mathrm{Si}_{9}$ and $\mathrm{Si}_{10}$ clusters were also investigated by the IR-UV twocolor ionization technique. ${ }^{38,39}$ These studies concentrated on structural assignment, so the IR spectra were recorded under fixed laser conditions. If the frequency of the IR light is in resonance with an IR active mode of the cluster, it can absorb one or more photons and heat up by subsequent internal vibrational energy redistribution. The increase in internal energy can enhance the ionization efficiency upon interaction with UV photons of a fixed energy as it may raise the total energy above the ionization threshold. The enhancement is a purely thermal effect as the clusters are irradiated with IR light well before interaction with the UV photons.

In the present experiments, the IR-UV two-color ionization efficiency is recorded for different conditions of the IR laser. It is not straightforward to measure the ionization probability from the experimental data alone, as the total intensities of the neutral clusters in the molecular beam are unknown. The intensities reported below where thus rescaled with respect to the abundance of $\mathrm{Si}_{8}$, which can serve as a reference signal, because its ionization energy ${ }^{51}$ of $7.46-7.87 \mathrm{eV}$ is below the single photon energy of the $\mathrm{F}_{2}$ gas laser. This implies that the quoted experimental ionization efficiencies of $\mathrm{Si}_{6}, \mathrm{Si}_{7}$ and $\mathrm{Si}_{10}$ are relative to the intensity of $\mathrm{Si}_{8}$, which is a good approximation if the abundances of the $\mathrm{Si}_{n}(n=6-10)$ clusters are similar.

Kinetic modeling of the IR-UV two-color ionization in silicon clusters uses quantum chemical ingredients obtained at the DFT/BPW91/6-311+G* level of theory. The ionization probability in the photoionization step was taken from Fowler. $^{52}$ This approach has previously been used in clusters physics, ${ }^{53}$ and it is notably expected to work in the present clusters for which the HOMO-LUMO gap is significant. ${ }^{54}$ Given the (vertical) ionization energy $\Phi_{0}$, also obtained from the DFT calculation, the photoionization yield $Y$ is expressed in this approach as

$$
\ln \left(\frac{Y}{[T(E)]^{2}}\right)=\Gamma+\ln f\left(\frac{h \nu-\Phi_{0}}{k_{\mathrm{B}} T(E)}\right),
$$

where $\Gamma$ is an arbitrary constant taken as $10^{7}$ atomic units, $E$ the internal energy and $h \nu$ the photon energy. For the clusters presently investigated $\left(\mathrm{Si}_{6}, \mathrm{Si}_{7}\right.$ and $\left.\mathrm{Si}_{10}\right)$, the ionization energy only slightly exceeds the $7.9 \mathrm{eV}$ UV photon energy, so the function $f$ can be approximated as ${ }^{52}$

$$
f(x)=\sum_{k \geq 0} \frac{(-1)^{k+1}}{k^{2}} \mathrm{e}^{k x} .
$$

The internal energy $E$ in the kMC trajectories is monitored as a function of time, and the statistical temperature $T(E)$ is determined from the microcanonical density of states $\Omega(E)$ through its definition, $1 / T(E)=\partial \ln \Omega(E) / k_{\mathrm{B}} \partial E$. Physically, the above 

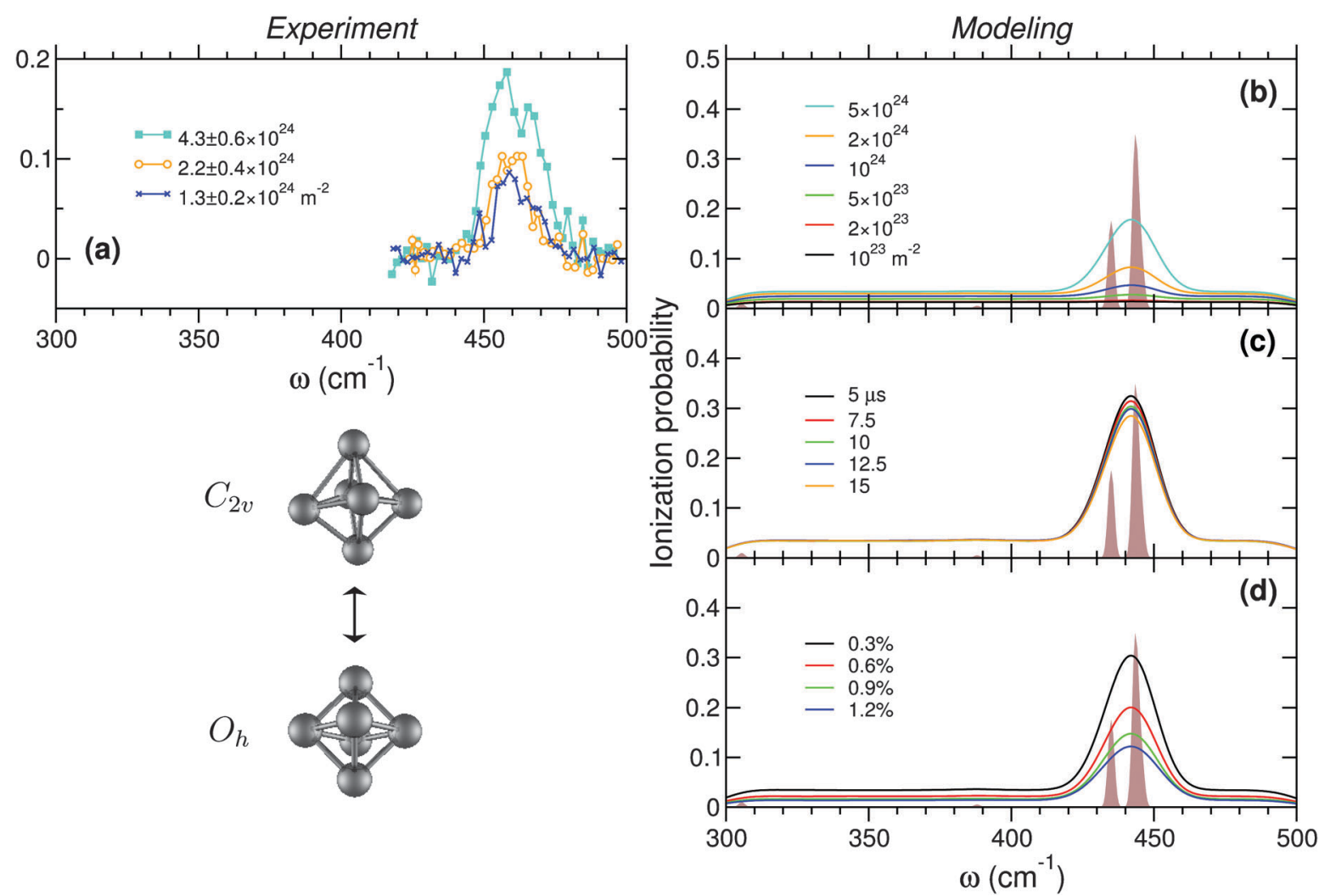

Fig. 2 Ionization probability of $\mathrm{Si}_{6}$ as a function of wavenumber, measured experimentally (left panel) and obtained from modeling (right panels) for different IR laser conditions. In (a) and (b) the photon exposure is varied for a macropulse duration of $8 \mu \mathrm{s}(10 \mu \mathrm{s}$ in the modeling) and an excitation laser bandwidth of $0.3 \%$; in (c) the macropulse duration is varied at fixed photon exposure of $5 \times 10^{24} \mathrm{~m}^{-2}$; in (d) the IR laser bandwidth is varied while the photon exposure is constant at $5 \times 10^{24} \mathrm{~m}^{-2}$ and for a macropulse duration of $10 \mu \mathrm{s}$. The theoretical linear absorption spectrum at equilibrium (110 $\mathrm{K}$ ) is superimposed on the calculated action spectra as brown histograms.

formulas convey the effect of the IR laser that raises the ionization yield through an increase in temperature. Here $t_{\mathrm{obs}}=50 \mu \mathrm{s}$ is taken as the time at which the UV laser pulse is applied.

The measured ionization probability of $\mathrm{Si}_{6}$ clusters is represented in Fig. 2(a) as a function of wavenumber and for different photon exposures. In the modeling we also considered separately the effects of varying macropulse durations and laser bandwidths, although these aspects were not addressed experimentally for $\mathrm{Si}_{6}$. The resulting spectra are shown in Fig. 2(b-d). Here we should stress that, in absence of any accurate guess for $\Gamma$ the magnitudes of the calculated ionization probabilities could be off by some factor relative to the measurements. Moreover it is unclear whether the same value of this parameter should be used for all cluster sizes. The experimental spectra exhibit a single peak near $460 \mathrm{~cm}^{-1}$, in agreement with earlier measurements. ${ }^{38,39}$ As the photon exposure is increased, the maximum ionization efficiency increases and, to a lesser extent, the band also broadens (fwhm increases from $18 \mathrm{~cm}^{-1}$ to $25 \mathrm{~cm}^{-1}$ over the range of photon exposure). Although intuitively anticipated, no spectral shift can be discerned for this band. As noted earlier, the experiment is compatible with two structural isomers with very similar IR signatures, ${ }^{38,45}$ known to interconnect with each other. ${ }^{55}$ The most stable isomer found by the present DFT method has $C_{2 \mathrm{v}}$ symmetry but lies very close to the $O_{\mathrm{h}}$ structure. The two minima are connected by a low energy barrier. Further computational evidence for such a low barrier is provided by the existence of a very low normal frequency close to $17 \mathrm{~cm}^{-1}$. The linear absorption spectrum of the $C_{2 \mathrm{v}}$ minimum at $110 \mathrm{~K}$ shows two main peaks near 435 and $445 \mathrm{~cm}^{-1}$, which after accounting for rotational broadening yield a single broader peak centered close to $440 \mathrm{~cm}^{-1}$. This value is about $3 \%$ lower than the experimental $460 \mathrm{~cm}^{-1}$. The simulations thus do quite little to shift the multiple photon resonance towards the experimental band, suggesting that only few IR photons are required for observing this band. The modeled band can still be brought into agreement with the experimental one if a scaling factor of 1.03 is used, consistently with earlier calculations using the BP86 functional. ${ }^{38,39}$

As in the experiment, the modeling [Fig. 2(b)] shows that a higher photon exposure leads to a higher intensity and the absolute ionization probabilities are also similar in magnitude. The comparable maximum ionization probabilities (about 20\%) found in the experiment and in the modeling under comparable conditions validate the value chosen for the prefactor $\Gamma$. Also similar to the experiment, no visible shift in the band position can be noted but a visible broadening as exposure is increased (30 to $48 \mathrm{~cm}^{-1}$ ). One minor discrepancy is the slight asymmetry 
in the band, whereas the calculations predict essentially Gaussian symmetric shapes. We tend to attribute this discrepancy to the Gaussian convolution that we use to account for rotational broadening, but also possibly to the non-symmetric lineshape of the free-electron laser. In addition to the variation of the photon exposure, we have also varied in the modeling the macropulse duration, while keeping the total photon exposure constant at a value of $5 \times 10^{24} \mathrm{~m}^{-2}$. At this same exposure and a pulse duration of $10 \mu \mathrm{s}$, the bandwidth was also varied. The resulting ionization spectra, shown in Fig. 2(c) and (d), exhibit only modest variations with some slight broadening and a loss in intensity as the bandwidth increases. The lower ionization probability at larger bandwidths was expected, because a smaller fraction of the clusters are resonant in this case. Repeating the calculations far below the near-saturation regime produces similar results, namely both parameters have a limited influence on the action spectrum.

Broader ranges of experimental conditions were explored for $\mathrm{Si}_{7}$. The experimental and modeled ionization probabilities of $\mathrm{Si}_{7}$ under different conditions are represented in Fig. 3. The photon exposure [Fig. 3(a) and (d)], the macropulse duration under constant photon exposure [Fig. 3(b) and (e)] and the laser bandwidth [Fig. 3(c) and (f)], were respectively varied.

The results at varying photon fluence are consistent with those obtained for $\mathrm{Si}_{6}$, with a clear increase in the overall signal but no band shift. For this cluster, increasing the pulse duration has also a very limited effect, but some broadening and minor intensity loss occurs for increasing bandwidth. The predictions of our modeling are all consistent with those measurements. At the harmonic level, the current DFT calculations predict a main absorption peak near $407 \mathrm{~cm}^{-1}$, but accounting for anharmonicities in the VPT2 approach shifts the peak slightly to the red. Interestingly, the modeled ionization spectra obtained here are not red shifted, buy slightly blue shifted with respect to the linear absorption spectra, which is consistent with the experiment. The blueshift is a manifestation of specific dynamical anharmonicities resulting from higher nonlinear effects occurring as the cluster temperature increases upon photon absorption. The modeled shift is, however, not sufficient to reach a complete experimental agreement.

Additional experimental and computed ionization spectra for the $\mathrm{Si}_{10}$ cluster are reported as ESI. $\dagger$ The experimental results are very similar to those obtained for $\mathrm{Si}_{7}$. The modeled IR absorption spectrum exhibits several peaks in the same spectral range, but it is found that only the most intense peaks contribute significantly to the action spectrum. In the model, another band located near $320-330 \mathrm{~cm}^{-1}$ only becomes visible at a photon exposure of approximately $6 \times 10^{24} \mathrm{~m}^{-2}$. However, this band is not visible in the experiment.


Fig. 3 Ionization probability of $\mathrm{Si}_{7}$ as a function of wavenumber, measured experimentally (left panels) and obtained from modeling (right panels) for different IR laser conditions. In (a) and (d) the photon exposure is varied for a macropulse duration of $10 \mu \mathrm{s}$; in (b) and (e) the macropulse duration is varied at fixed photon exposures of $3.8 \times 10^{24} \mathrm{~m}^{-2}$ (experiment) or $5 \times 10^{24} \mathrm{~m}^{-2}$ (modeling); in (c) and (f) the IR laser bandwidth is varied while the photon exposures are constant at $6.3 \times 10^{24} \mathrm{~m}^{-2}$ (experiment) or $5 \times 10^{24} \mathrm{~m}^{-2}$ (modeling) and for a macropulse duration of $10 \mu \mathrm{s}$. The theoretical linear absorption spectrum at equilibrium is superimposed on the calculated action spectra as brown histograms. 




Fig. 4 Average number of IR photons absorbed by $\mathrm{Si}_{n}$ clusters obtained by modeling under a photon exposure of $5 \times 10^{24} \mathrm{~m}^{-2}$, a macropulse duration of $10 \mu \mathrm{s}$, and a $0.3 \%$ bandwidth of the IR laser, as a function of the wavenumber of the excitation.

The modeling provides additional information about the mechanisms of IR-UV two-color ionization through quantities that are hardly accessible experimentally. We show in Fig. 4 the average number of absorbed IR photons when $\mathrm{Si}_{6}, \mathrm{Si}_{7}$ and $\mathrm{Si}_{10}$ are exposed to the UV laser. The nonzero baseline and fractional numbers obtained away from resonances are associated with stimulated emission of occasionally absorbed photons. Except for this baseline, the variations of those numbers with laser wavelength reflect those of the action spectrum, but the variations in intensity are generally much stronger. This effect arises due to the highly nonlinear character of the photoionization rate, whose dependence on internal energy (and the number of absorbed photons) is extremely strong in the Fowler description. ${ }^{52}$ The variations are also rather rounded near the value of one single photon, which reflects the highly nonlinear character of the photon absorption efficiency. It should also be noted that in contrast with the action spectra no rotational broadening is accounted for in Fig. 4. The average number of absorbed photons usually remains close to or below one, keeping the system below the statistical UV-induced ionization threshold. As expected, occasional absorption of more than one photon only occurs near resonance, and under the present conditions reaches a maximum absorption of 3-5 photons. The observation that so few photons have already a significant effect on the photoionization intensity is an indication of the high sensitivity of the IR-UV two-color ionization spectroscopic technique.

\section{Multiphoton dissociation of vanadium oxide clusters}

Vanadium oxide clusters are promising candidates as nanocatalysts $^{56-58}$ and as such the relation between their structure and reactivity has been scrutinized. ${ }^{59,60}$ Their vibrational properties are now well documented owing to a number of cluster-rare gas photodissociation $^{10,40,61,62}$ and IR-MPD ${ }^{63,64}$ investigations, usually supported by DFT calculations in the harmonic approximation.

Here the IR spectra of $\mathrm{V}_{4} \mathrm{O}_{10}{ }^{+}$and $\mathrm{V}_{3} \mathrm{O}_{7}{ }^{+}$were measured using the laser-induced dissociation of the bare cluster and of the cluster-Xe rare gas complex, respectively. Dissociation of $\mathrm{V}_{4} \mathrm{O}_{10}{ }^{+}$proceeds through the channel $\mathrm{V}_{4} \mathrm{O}_{8}{ }^{+}+\mathrm{O}_{2}$. The ingredients necessary to the kinetic modeling again originate from DFT calculations. In order to account for dispersion forces that are relevant in the cluster-xenon complex, the $\mathrm{wB} 97 \mathrm{xD}$ functional was chosen. The QZVP and TZVP basis sets were employed in all-electron calculations for $\mathrm{V}_{3} \mathrm{O}_{7}{ }^{+}(+\mathrm{Xe})$ and $\mathrm{V}_{4} \mathrm{O}_{10}{ }^{+}$, respectively. The dissociation rates $k$ were evaluated as a function of internal energy $E$ using the simple Rice-Ramsperger-Kassel (RRK) theory, ${ }^{65}$

$$
k_{\mathrm{RRK}}(E)=\nu_{0}\left(1-\frac{D}{E}\right)^{\kappa-1}
$$

where the prefactor $\nu_{0}$ is a typical vibrational constant taken as $10^{12} \mathrm{~s}^{-1}, D$ is the dissociation energy, and $\kappa$ the number of degrees of freedom of the parent cluster. Here $D$ refers to the fragmentation of $\mathrm{V}_{4} \mathrm{O}_{10}{ }^{+}$into $\mathrm{V}_{4} \mathrm{O}_{8}{ }^{+}+\mathrm{O}_{2}$ or to the evaporation of the xenon atom from $\mathrm{V}_{3} \mathrm{O}_{7}{ }^{+}-\mathrm{Xe}$. The appropriate values for the two clusters are given as ESI. $\dagger$

Fig. 5 shows the variations of the dissociation probability of $\mathrm{V}_{4} \mathrm{O}_{10}{ }^{+}$into $\mathrm{V}_{4} \mathrm{O}_{8}{ }^{+}+\mathrm{O}_{2}$ for different conditions of the IR laser. As was the case for silicon clusters, the comparable magnitudes in the dissociation probability between experiment and modeling indicates that the only adjustable parameter in our calculations (the prefactor $\nu_{0}$ ) was reasonably chosen. Increasing the photon exposure, the experimental spectra of Fig. 5(a) display a higher dissociation propensity as well as some broadening associated with a small blueshifting of the main absorption peak near $1050 \mathrm{~cm}^{-1}$. In the modeling, the band probed here is overestimated by about $50 \mathrm{~cm}^{-1}$, which could have been accounted for by a significant scaling factor close to 0.92 in conventional harmonic DFT calculations. ${ }^{11}$ It actually comprises three similar vanadyl stretches that differ from each other owing to the symmetry breaking in the cationic cluster, the fourth vanadyl band lying near $750 \mathrm{~cm}^{-1}$ upon electron removal from the HOMO of the neutral $T_{\mathrm{d}}$ symmetric cluster $\mathrm{V}_{4} \mathrm{O}_{10}$. In principle, much of the discrepancy between the experimental and modelled vibration mode should be addressed by the anharmonic treatment. Indeed the anharmonic lines are redshifted relative to (unscaled) harmonic calculations (see ESI $\dagger$ ), yet the effect is rather limited and the VPT2 approach employed here appears clearly insufficient. The simulated IR-MPD spectrum shows an additional slight redshift relative to the linear absorption spectra, which again emphasizes the specific dynamical anharmonicities involved in those nonlinear spectra and the higher temperatures reached by the system exposed to the resonant laser.

Besides this absolute spectral shift, the correspondence of experiments and the modeling turns out to be quite good: the absolute dissociation probability, its increase with increasing photon exposure, the broadening and the blue shift of the absorption bands with increased photon exposure, are all reproduced. 

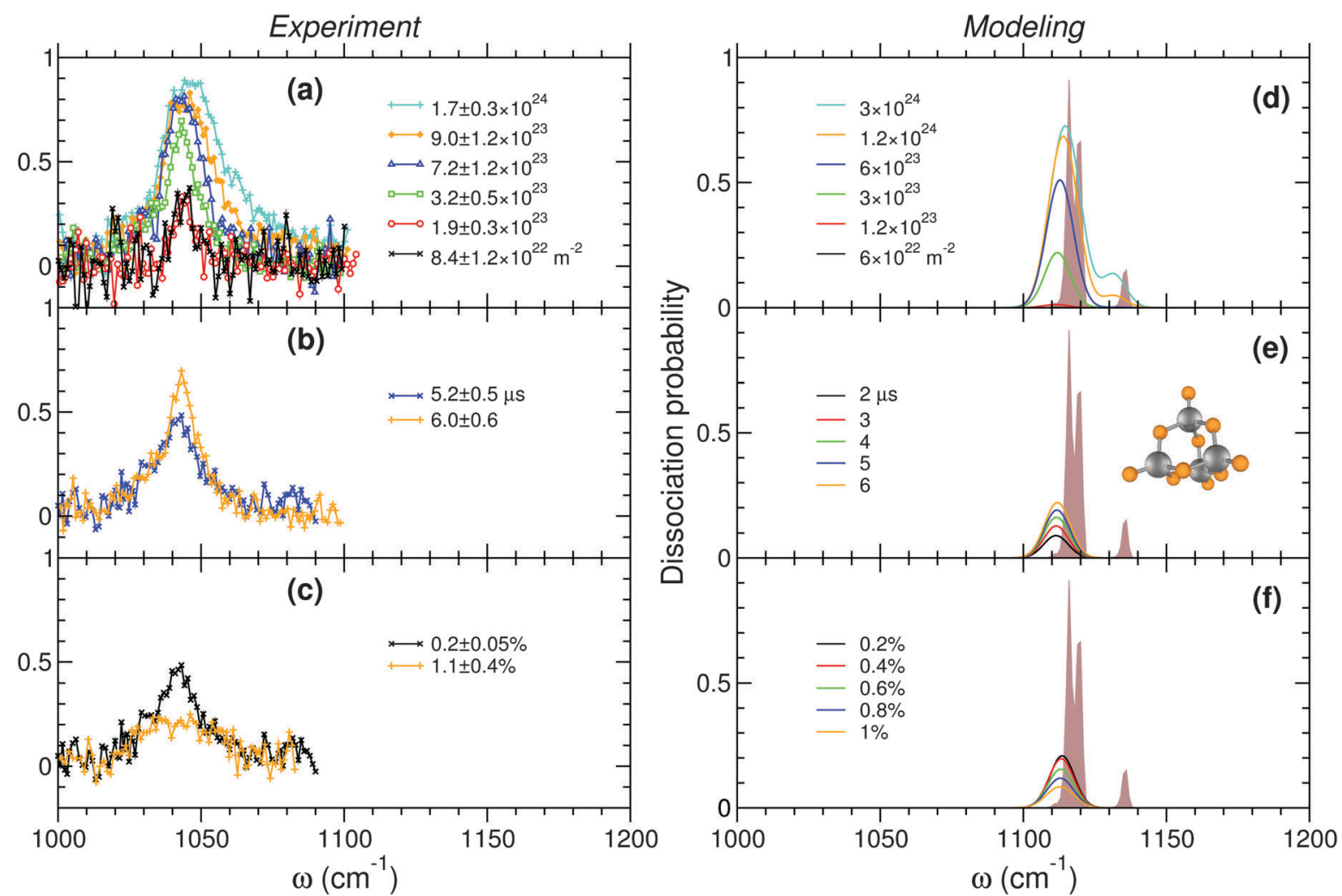

Fig. 5 Dissociation probability of $\mathrm{V}_{4} \mathrm{O}_{10}{ }^{+}$as a function of wavenumber, measured experimentally (left panels) and obtained from modeling (right panels) for different conditions of excitation. In (a) and (d) the photon exposure is varied for a macropulse duration of $6 \mu$ s; in (b) and (e) the macropulse duration is varied at fixed photon exposures of $3.2 \times 10^{23} \mathrm{~m}^{-2}$ (experiment) and $3 \times 10^{23} \mathrm{~m}^{-2}$ (modeling); in (c) and (f) the IR laser bandwidth is varied while the photon exposures are constant with the same values as in panels (b) and (e), respectively, the macropulse duration being fixed at $5.2 \mu \mathrm{s}$ (experiment) and $6 \mu$ s (modeling). The theoretical linear absorption spectrum at equilibrium is superimposed on the calculated action spectra as brown histograms.

Clearly, the most apparent disagreement lies in the faster reaching of saturation in the experiment, which relates to inaccuracies in the quantum chemical IR intensities and the dipole moment surface. The origin of the blue shift and spectral broadening can be traced back to the increasing response of the higher frequency peak near $1135 \mathrm{~cm}^{-1}$ in Fig. 5(d-f), which is characteristic of the less active but higher frequency $\mathrm{V}=\mathrm{O}$ vanadyl mode. The two $\mathrm{V}=\mathrm{O}$ modes near $1120 \mathrm{~cm}^{-1}$ merge in the action spectrum once rotational broadening is accounted for. As the photon exposure is increased, the three vanadyl modes eventually merge, producing the high frequency shoulder in the calculated spectrum, consistent with the experiment.

Upon increasing the macropulse duration at an approximately constant photon exposure, a slight increase in the dissociation probability is observed [see Fig. 5(b)]. Similar variations are also found in the calculated spectra in Fig. 5(e), though in a regime further away from saturation. This could indicate that the IR-MPD process is not sufficiently efficient when the photons are delivered to the system at higher rates. They are not adequately absorbed due to the anharmonic bottleneck. In such situations the assumption that IVR is complete between two successive events may become questionable. Increasing the bandwidth significantly broadens the experimental peak in Fig. 5(c) and diminishes its intensity, similarly as previous observations on neutral silicon clusters.
The broadening effect is much less marked in the modeling, however the decrease in intensity is reproduced.

The experimental IR photodissociation spectrum of the xenon-tagged $\mathrm{V}_{3} \mathrm{O}_{7}{ }^{+}$cluster had to be corrected for contamination related to the dissociation of $\mathrm{V}_{3} \mathrm{O}_{7}{ }^{+}-\mathrm{Xe}_{2}$, which has an intensity comparable to that of $\mathrm{V}_{3} \mathrm{O}_{7}{ }^{+}$-Xe. In attempting to model the dissociation of the xenon messenger atom from $\mathrm{V}_{3} \mathrm{O}_{7}{ }^{+}-\mathrm{Xe}$, the couplings matrix associated with the tagged complex could not be exploited for the modeling due to excessive anharmonicities likely associated to the rare gas atom being too weakly bound to the cluster. As a first approximation, it was assumed that the anharmonicities of the vibrational modes in the mid-IR range were only little affected by the presence of the Xe messenger atom, whose binding modes have lower frequencies (see the full list of normal mode frequencies in the $\mathrm{ESI} \dagger)$.

The experimental and calculated spectra of $\mathrm{V}_{3} \mathrm{O}_{7}{ }^{+}-\mathrm{Xe}$ obtained under different laser conditions are shown in Fig. 6. For this system the experimental spectra display two bands near $1030-1050 \mathrm{~cm}^{-1}$. For the 'ring' isomer, which is the lowest-energy conformer upon attaching a xenon atom, the DFT calculations find three $\mathrm{V}=\mathrm{O}$ vanadyl bands, two near-degenerate and more intense than a third higher-frequency mode (see ESI $\dagger$ ). Those results are also rather close to earlier calculations of Asmis and Sauer on the related $\mathrm{V}_{3} \mathrm{O}_{7}{ }^{+}-\mathrm{Ar}$ complex. ${ }^{11}$ This near-degeneracy is 

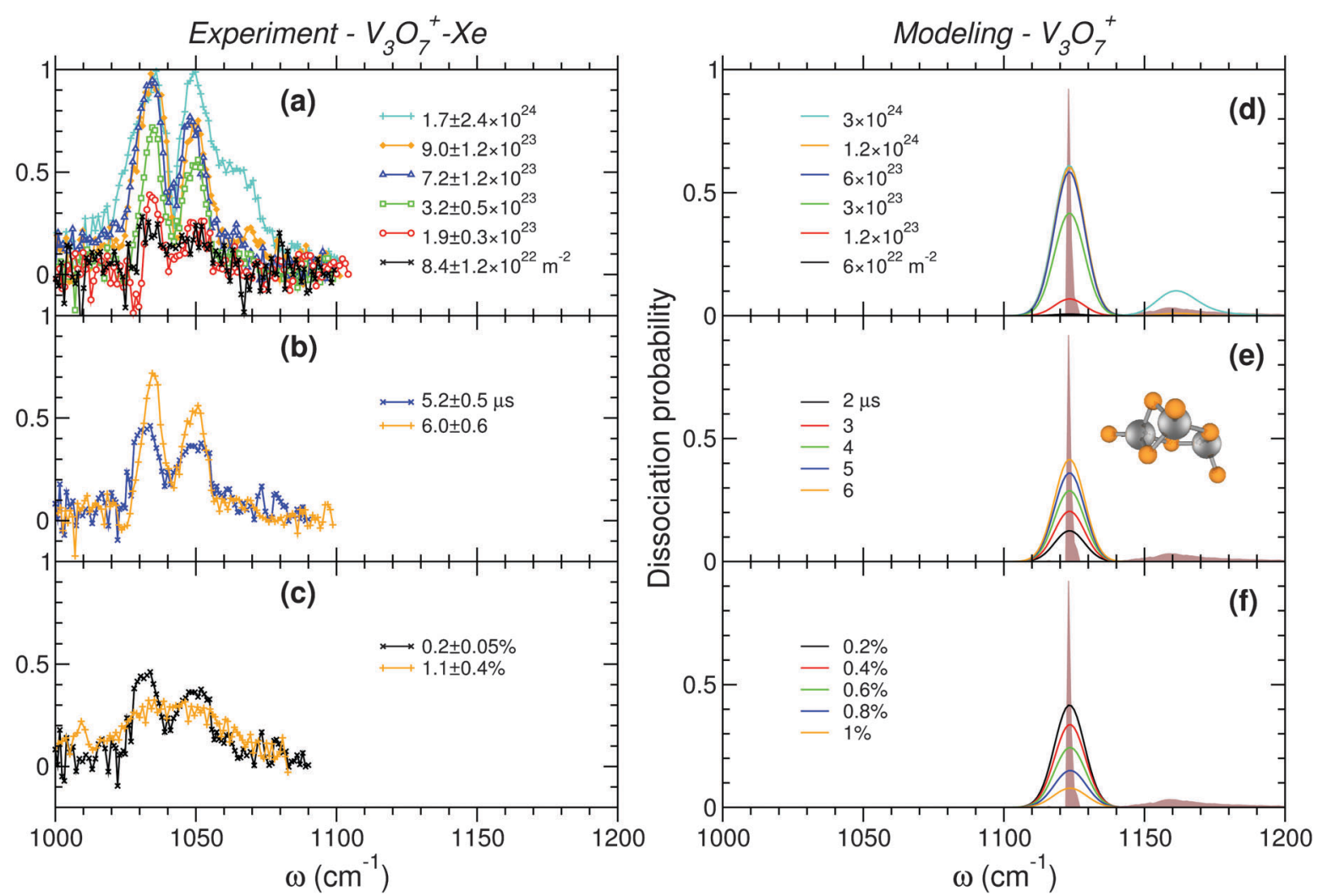

Fig. 6 Dissociation probability of the xenon atom from $\mathrm{V}_{3} \mathrm{O}_{7}{ }^{+}+\mathrm{Xe}$ as a function of wavenumber, measured experimentally (left panels) and obtained from modeling (right panels) for different conditions of excitation. In (a) and (d) the photon exposure is varied for a macropulse duration of $6 \mu$ s; in (b) and (e) the macropulse duration duration is varied at fixed photon exposures of $3.2 \times 10^{23} \mathrm{~m}^{-2}$ (experiment) and $3 \times 10^{23}$ (modeling); in (c) and (f) the IR laser bandwidth is varied while the photon exposures are constant with the same values as in panels (b) and (e), respectively, the macropulse duration being fixed at $5.2 \mu$ s (experiment) and $6 \mu$ s (modeling). The theoretical linear absorption spectrum at equilibrium is superimposed on the calculated action spectra as brown histograms.

not lifted by the anharmonic calculations. Besides a global blueshifting that would require additional scaling factors, the linear absorption spectrum obtained at $170 \mathrm{~K}$ (for the bare cluster) differs from the experimental spectra mostly in the relative intensity of the two peaks. The discrepancy could result from a poor accuracy in predicting the photon absorption efficiency in presence of the xenon atom.

The measured dissociation spectrum of $\mathrm{V}_{3} \mathrm{O}_{7}{ }^{+}$-Xe exhibits similar trends as the IR-MPD spectra of $\mathrm{V}_{4} \mathrm{O}_{10}{ }^{+}$when the laser conditions are varied, with a general increase, broadening, and blue shifting of the two-peak structure as the photon exposure is increased. Increasing the macropulse duration under constant photon exposure or the bandwidth [Fig. 6(b and c)] has similar consequences as for $\mathrm{V}_{4} \mathrm{O}_{10}{ }^{+}$. These trends are satisfactorily reproduced by the modeling. As was the case for $\mathrm{V}_{4} \mathrm{O}_{10}{ }^{+}$, a shoulder on the blue side is noted in the measurements at high photon exposure. A similar interpretation as for $\mathrm{V}_{4} \mathrm{O}_{10}{ }^{+}$would require the presence of three bands, the highest-frequency mode emerging only at sufficiently high photon exposures. The similar spacing between the two experimental bands at 1035 and $1068 \mathrm{~cm}^{-1}$ and the calculated peaks at 1124 and $1160 \mathrm{~cm}^{-1}$ suggests that the bands near 1035 and $1050 \mathrm{~cm}^{-1}$ in the experimental spectrum correspond to the degenerate modes of the peak computed at $1124 \mathrm{~cm}^{-1}$ whose IR intensity is less accurately predicted. The harmonic calculations in presence of the xenon messenger (Table S5 in the ESI $\dagger$ ) indeed confirm the existence of two such modes with comparable intensities, as well as a higher frequency and less intense mode. A second possible explanation may come from other possible isomers stabilized by the xenon atom, as was already reported for the similar argon-tagged $\mathrm{V}_{3} \mathrm{O}_{7}{ }^{+}$cluster. ${ }^{11}$ For the present xenon-tagged cluster, a similar process is predicted from DFT calculations with a 'cage' isomer being more stable in absence of the tagging atom. However, this isomer does not show a multiple peak spectrum in the present spectral range (see ESI $\dagger$ ). Third, it is also possible that yet a different isomer could arise only at high temperatures that are reachable under high photon fluences. Clearly a more complete interpretation of those intriguing nonlinear effects would require additional and dedicated efforts. In comparison with the major influence of laser fluence, the effects of the macropulse duration and laser bandwidth appear qualitatively similar but quantitatively less accurate. We attribute this difference to the lower intensities obtained in the modeling under the photon exposure of $3 \times 10^{23} \mathrm{~m}^{-2}$ with respect to experimental spectra. Such a discrepancy between the saturation regimes in the experiment and the modeling could also be noted for the $\mathrm{V}_{4} \mathrm{O}_{10}{ }^{+}$system. 


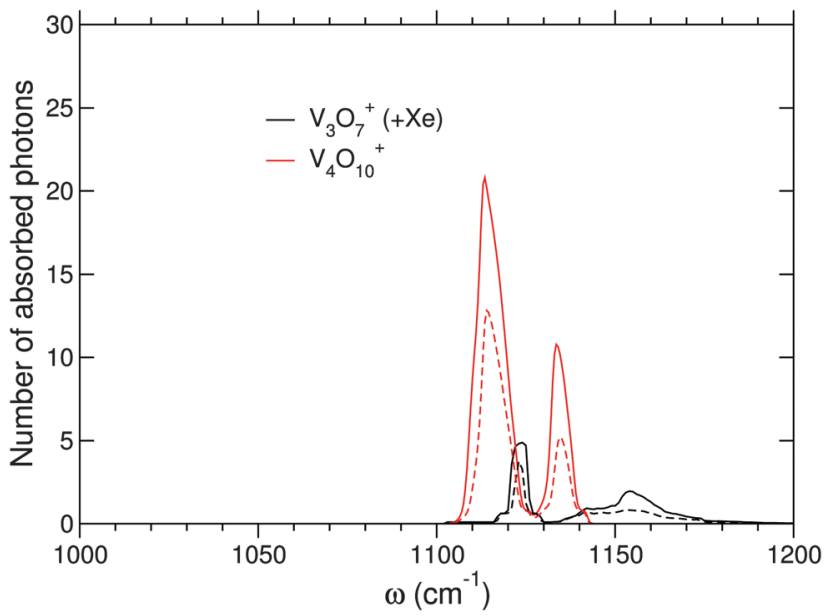

Fig. 7 Average number of photons absorbed by vanadium oxide clusters obtained by modeling under a photon exposure of $3 \times 10^{23} \mathrm{~m}^{2} \mathrm{~s}$ (dashed lines) and $3 \times 10^{24} \mathrm{~m}^{2} \mathrm{~s}$ (solid lines), a macropulse duration of $6 \mu \mathrm{s}$, and a $0.2 \%$ bandwidth, as a function of the wavenumber of the excitation.

As for neutral silicon clusters, the average numbers of photons absorbed by the two cationic clusters before they dissociate or evaporate the messenger atom were evaluated for typical laser conditions of two photon exposures of $3 \times 10^{23} \mathrm{~m}^{-2}$ and $3 \times 10^{24} \mathrm{~m}^{-2}$, and a macropulse duration of $6 \mu \mathrm{s}$. Their variations with the wavenumber of the excitation are depicted in Fig. 7. The numbers of absorbed photons are rather high for both clusters, and as expected the IR-MPD spectrum of $\mathrm{V}_{4} \mathrm{O}_{10}{ }^{+}$is indeed associated with several tens of IR photons near resonance, even below the saturation regime. However, the shoulder on the blue side of the main band in the experimental spectrum of this cluster is created by about a factor of two less photons. This result is consistent with the need for high fluences to observe the band. For the xenontagged $\mathrm{V}_{3} \mathrm{O}_{7}{ }^{+}$cluster, approximately five photons are needed to induce the Xe evaporation under conditions similar to the experiment. Nonlinear effects are also manifested on the numbers of photons obtained for the different bands, which appear to scale differently with photon exposure.

\section{Discussion and concluding remarks}

Although the two types of systems investigated in the present work differ in their intrinsic chemical properties and in the way they were experimentally addressed, the vibrational action spectra show general trends with varying laser conditions that reveal the nonlinear character of action spectroscopy techniques. Increases in signal intensity with increasing photon fluence or macropulse duration under fixed photon exposure are noteworthy observations that naturally convey the greater propensity for absorption and the stronger molecular response. Spectral broadenings were also commonly found but appear as secondary effects. In the case of vanadium oxide clusters, they could be interpreted as due to the emergence of higher frequency peaks that broaden and merge with the main peaks at sufficiently high photon exposures. In contrast with those clearly evidenced effects, spectral shifts were not found to be very significant for the present systems. This is at variance with hydrocarbon molecules for which additional redshifts due to dynamical anharmonicities were identified. ${ }^{35,36}$

With essentially no adjustable parameters, our kinetic modeling of the different experiments generally lead to a quite satisfactory description of the nonlinear effects. The main discrepancies probably lie in the residual offset of the spectra, which appear red- and blueshifted for neutral silicon and cationic vanadium oxide clusters, respectively, reflecting the inherent errors of the quantum chemistry data used in the modeling. Obviously, the usual scaling procedure employed to correct harmonic spectra could have been applied as well here. However, given that structural assignment has already been achieved for those clusters based on earlier DFT investigations, ${ }^{11,38,39}$ it was not the purpose of the present work to repeat those efforts, but rather to focus on higher-order nonlinear processes.

It is fair to recognize that, despite overall agreement, some experimental trends were not strictly reproduced by our modeling in the case of vanadium oxide clusters, notably in the description of saturation regimes and their dependence on photon exposure. A second notable disagreement was obtained for the xenontagged $\mathrm{V}_{3} \mathrm{O}_{7}{ }^{+}$cluster, which we were not able to reliably describe owing to the breakdown in the second-order perturbation theory approximation for this highly anharmonic system. At this stage, the few quantum chemical calculations performed for this cluster do not fully satisfactorily explain the two-peak vibrational structure found in the experiment and the emergence of a shoulder on the blue side at high fluences, although all trends in the nonlinear effects are otherwise well reproduced. Different possible causes were proposed for this discrepancy, including the contribution of other isomers especially at higher temperatures obtained after exposure to the laser. However, it is also likely that the VPT2 approach would not be sufficient to describe these other isomers and their anharmonicities, which sets some limits on the present kinetic approach for dissociation spectroscopy of cluster-rare gas complexes.

One approximation on the modeling was the neglect of the micropulse structure, whose description would have entailed extremely short time resolutions and poor statistics. Experimental macropulses of $10 \mu \mathrm{s}$ duration typically contain tens of thousands micropulses separated by $1 \mathrm{~ns}$. The present modeling indicates that, even under high photon exposures, only a limited number of photons are absorbed at maximum intensity, suggesting that the probability of multiple photon absorption under exposure to a single micropulse is unlikely. In order to address this issue in greater details, it would be better to use an atomistic approach where the molecular dynamics of the cluster exposed to the laser field is explicitly simulated. ${ }^{34}$ One requirement for this alternative approach is the development of suitable potential energy and electric dipole moment surfaces, and for such oxides simple ionic potentials (BornMayer + Coulomb, possibly improved for polarization effects) would probably enable such molecular simulations. One issue related to the neglect of the micropulse structure is the residual 
dependence on the pulse length at fixed total photon exposure in the modeling, which clearly points to dynamical anharmonicities and the heating of the system under laser exposure.

The non-uniform profile of the actual laser beam was also neglected in our modeling. This approximation makes a strict comparison with experiment more difficult, but could conveniently be overcome by introducing this spatial distribution, randomly selecting the photon exposure accordingly at the beginning of each trajectory. This would involve additional statistical averaging and a far greater number of individual kMC trajectories, but could be attempted for a more direct comparison. Finally, another approximation found to be of significance notably in the case of silicon clusters is that of rotational broadening, which for simplicity was performed as a Gaussian convolution with appropriate width and likely contributes to excessively symmetric lineshapes in the modeling. ${ }^{44}$ It is possible to take the entire rovibrational structure into account in the modeling, as was achieved in the case of finite temperature IR spectra. ${ }^{66}$ Extension to action spectra is straightforward in principle, and it would be useful to check the present results against such more refined models. This would also cancel the need for applying the rotational broadening at a fixed temperature, and instead would let those effects vary with internal energy along the multiphoton absorption process.

Despite those limitations and the various possible improvements, the quality of overall agreement indicates that the essential ingredients causing the nonlinear effects are correctly captured by the kMC model. The present results should motivate further work on related systems for which multiphoton dissociation have already been obtained such as aluminum oxide clusters. ${ }^{67,68}$ Beyond reproducing trends, one should also aim at characterizing the spectral features on more quantitative grounds, directly comparing peak positions, broadenings and intensities. In view of the present encouraging results, those goals seem now at hand.

\section{Acknowledgements}

We gratefully acknowledge the Stichting voor Fundamenteel Onderzoek der Materie (FOM) for the support of the FELIX Laboratory. The research leading to these results has received funding from the European Community's Seventh Framework Programme (FP7/2007-2013) under grand agreement no. 312284. This work is supported by the Research Foundation-Flanders (FWO), the KU Leuven Research Council (GOA/14/007). F.C. and E.J. acknowledge support from the bilateral Tournesol exchange program (T2014.13/30977YG). D.K. acknowledges support from the Netherlands Organisation for Scientific Research (NWO) as part of the Dutch Astrochemical Network.

\section{References}

1 T. S. Zwier, Annu. Rev. Phys. Chem., 1996, 47, 205.

2 M. A. Duncan, Int. Rev. Phys. Chem., 2003, 22, 407.

3 D. Oepts, A. G. F. van der Meer and P. W. van Amersfoort, Infrared Phys. Technol., 1995, 36, 297-308.
4 J. Lemaire, P. Boissel, M. Heninger, G. Mauclaire, G. Bellec, H. Mestdagh, A. Simon, S. Le Caer, J. M. Ortega, F. Glotin and P. Maître, Phys. Rev. Lett., 2002, 89, 273002.

5 C. Kapota, J. Lemaire, P. Maître and G. Ohanessian, J. Am. Chem. Soc., 2004, 126, 1836.

6 M. Rossi, V. Blum, P. Kupser, G. von Helden, F. Bierau, K. Pagel, G. Meijer and M. Scheffler, J. Phys. Chem. Lett., 2010, 1, 3465.

7 O. Asvany, P. P. Kumar, B. Redlich, I. Hegemann, S. Schlemmer and D. Marx, Science, 2005, 309, 1219.

8 K. Kwapien, M. Sierka, J. Döbler, J. Sauer, M. Haertelt, A. Fielicke and G. Meijer, Angew. Chem., Int. Ed., 2011, 50, 1716.

9 M. A. Martin-Drumel, O. Pirali, C. Falvo, P. Parneix, A. Gamboa, F. Calvo and Ph. Bréchignac, Phys. Chem. Chem. Phys., 2014, 16, 22062-22072.

10 A. Fielicke, R. Mitrić, G. Meijer, V. Bonačić-Koutecký and G. von Helden, J. Am. Chem. Soc., 2003, 125, 15716-15717.

11 K. R. Asmis and J. Sauer, Mass Spectrom. Rev., 2007, 26, 542-562.

12 A. Castro, M. A. L. Marques, J. A. Alonso and A. Rubio, J. Comput. Theor. Nanosci., 2005, 1, 231-255.

13 S. M. Lang, P. Claes, N. T. Cuong, M. T. Nguyen, P. Lievens and E. Janssens, J. Chem. Phys., 2011, 135, 224305.

14 R. W. Burgess and V. J. Keast, J. Phys. Chem. C, 2014, 118, 3194.

15 J. A. Pople, H. B. Schlegel, R. Krishnan, D. J. DeFrees, J. S. Binkley, M. J. Frisch, R. A. Whiteside, R. F. Hout and W. J. Hehre, Int. J. Quantum Chem., 1981, 15, 269.

16 R. F. Hout, B. A. Levi and W. J. Hehre, J. Comput. Chem., 1982, 3, 234.

17 P. Pulay, G. Fogarasi, G. Pongor, J. E. Boggs and A. Vargha, J. Am. Chem. Soc., 1983, 105, 7037.

18 D. J. DeFrees and A. D. McLean, J. Chem. Phys., 1985, 82, 333.

19 A. P. Scott and L. Radom, J. Phys. Chem., 1996, 100, 16502.

20 V. Barone, J. Chem. Phys., 2005, 122, 014108.

21 J. Bowman, K. M. Christoffel and F. Tobin, J. Phys. Chem., 1979, 83, 905-912.

22 R. Gerber and M. Ratner, Adv. Chem. Phys., 1988, 70, 97-132.

23 F. Fontaine-Vive, M. R. Johnson, G. J. Kearley, J. A. K. Howard and S. F. Parker, J. Am. Chem. Soc., 2006, 128, 2963.

24 M. Pavese, D. R. Berard and G. A. Voth, Chem. Phys. Lett., 1999, 300, 93.

25 X. Huang, S. Habershon and J. M. Bowman, Chem. Phys. Lett., 2003, 450, 253.

26 M.-P. Gaigeot, Phys. Chem. Chem. Phys., 2010, 12, 3336.

27 J. Banisaukas, J. Szczepanski, J. Eyler, M. Vala, S. Hirata, M. Head-Gordon, J. Oomens, G. Meijer and G. von Helden, J. Phys. Chem. A, 2003, 107, 782-793.

28 U. J. Lorenz, N. Solca, J. Lemaire, P. Maître and O. Dopfer, Angew. Chem., Int. Ed., 2007, 46, 6714.

29 C. G. Atkins, K. Rajabi, E. A. L. Gillis and T. D. Fridgen, J. Phys. Chem. A, 2008, 112, 10220.

30 J. Oomens, A. G. G. M. Tielens, B. G. Sartakov, G. von Helden and G. Meijer, Astrophys. J., 2003, 591, 968-985.

31 J. Oomens, B. G. Sartakov, G. Meijer and G. von Helden, Int. J. Mass Spectrom., 2006, 254, 1-19. 
32 A. Bekkerman, E. Kolodney, G. von Helden, B. Sartakov, D. van Heijnsbergen and G. Meijer, J. Chem. Phys., 2006, 124, 184312.

33 M. J. Nee, A. Osterwalder, D. M. Neumark, C. Kaposta, C. Cibrián Uhalte, T. Xie, A. Kaledin, J. M. Bowman, S. Carter and K. R. Asmis, J. Chem. Phys., 2004, 121, 7259.

34 F. Calvo and P. Parneix, ChemPhysChem, 2012, 13, 212-220.

35 P. Parneix, M. Basire and F. Calvo, J. Phys. Chem. A, 2013, 117, 3954-3959.

36 J. Oomens, A. J. A. van Roij, G. Meijer and G. von Helden, Astrophys. J., 2000, 542, 404-410.

37 P. Gruene, D. M. Rayner, B. Redlich, A. F. G. van der Meer, J. T. Lyon, G. Meijer and A. Fielicke, Science, 2008, 321, 674-676.

38 A. Fielicke, J. T. Lyon, M. Haertelt, G. Meijer, P. Claes, J. de Haeck and P. Lievens, J. Chem. Phys., 2009, 131, 177105.

39 M. Haertelt, J. T. Lyon, P. Claes, J. de Haeck, P. Lievens and A. Fielicke, J. Chem. Phys., 2012, 136, 064301.

40 K. R. Asmis, M. Brümmer, C. Kaposta, G. Santambrogio, G. von Helden, G. Meijer, K. Rademann and L. Wöste, Phys. Chem. Chem. Phys., 2002, 4, 1101-1104.

41 F. Wang and D. P. Landau, Phys. Rev. Lett., 2001, 86, 2050.

42 M. Basire, P. Parneix and F. Calvo, J. Chem. Phys., 2008, 129, 0811019.

43 M. Basire, P. Parneix, F. Calvo, T. Pino and P. Bréchignac, J. Phys. Chem. A, 2009, 113, 6947-6954.

44 P. Le Coupanec, D. Rouan and A. Léger, Astron. Astrophys., 1998, 338, 217.

45 E. C. Honea, A. Ogura, C. A. Murray, K. Raghavachari, W. O. Sprenger, M. F. Jarrold and W. L. Brown, Nature, 1993, 366, 42.

46 J. Müller, B. Liu, A. A. Shvartsburg, S. Ogut, J. R. Chelikowsky, K. W. M. Siu, K. M. Ho and G. Ganteför, Phys. Rev. Lett., 2000, 85, 1666.

47 G. Meloni, M. J. Ferguson, S. M. Sheehan and D. M. Neumark, Chem. Phys. Lett., 2004, 399, 389.

48 J. Bai, L. F. Cui, J. Wang, S. Soo, X. Li, J. Jellinek, C. Koehler, T. Frauenheim, L. S. Wang and X. C. Zeng, J. Phys. Chem. A, 2006, 110, 908.

49 R. R. Hudgins, M. Imai, M. F. Jarrold and P. Dugourd, J. Chem. Phys., 1999, 111, 7865.
50 J. T. Lyon, P. Gruene, A. Fielicke, G. Meijer, E. Janssens, P. Claes and P. Lievens, J. Am. Chem. Soc., 2009, 131, 1115.

51 K. Fuke, K. Tsukamoto, F. Misaizo and M. Sanekata, J. Chem. Phys., 1993, 99, 7807.

52 R. H. Fowler, Phys. Rev., 1931, 38, 45.

53 A. Prem and V. V. Kresin, Phys. Rev. A: At., Mol., Opt. Phys., 2012, 85, 025201.

54 J. Jalink, J. M. Bakker, T. Rasing and A. Kirilyuk, J. Phys. Chem. Lett., 2015, 6, 750-754.

55 A. D. Zdetsis, J. Chem. Phys., 2007, 127, 244308.

56 S. Feyel, J. Döbler, D. Schröder, J. Sauer and H. Schwarz, Angew. Chem., Int. Ed., 2006, 45, 4681.

57 F. Stavale, C. A. Achete and H. Niehus, Surf. Sci., 2007, 601, 4881-4887.

58 Y. S. Hu, X. Liu, J. O. Müller, R. Schlögl, J. Maier and D. S. Su, Angew. Chem., Int. Ed., 2009, 48, 210-214.

59 D. K. Böhme and H. Schwarz, Angew. Chem., Int. Ed., 2005, 44, 2336-2354.

60 T. Wende, J. Döbler, L. Jiang, P. Claes, E. Janssens, P. Lievens, G. Meijer, K. R. Asmis and J. Sauer, Int. J. Mass Spectrom., 2010, 297, 102-106.

61 M. Brümmer, C. Kaposta, G. Santambrogio and K. R. Asmis, J. Chem. Phys., 2003, 119, 12700-12703.

62 K. R. Asmis, G. Meijer, M. Brümmer, C. Kaposta, G. Santambrogio, L. Wöste and J. Sauer, J. Chem. Phys., 2004, 120, 6461-6470.

63 S. Feyel, H. Schwarz, D. Schröder, C. Daniel, H. Hartl, J. Döbler, J. Sauer, G. Santambrogio, L. Wöste and K. R. Asmis, ChemPhysChem, 2007, 8, 1640-1647.

64 G. Santambrogio, M. Brümmer, L. Wöste, J. Döbler, M. Sierka, J. Sauer, G. Meijer and K. R. Asmis, Phys. Chem. Chem. Phys., 2008, 10, 3992-4005.

65 (a) O. Rice and H. C. Ramsperger, J. Am. Chem. Soc., 1927, 49, 1617-1629; (b) L. S. Kassel, J. Phys. Chem., 1928, 32, 225-242.

66 F. Calvo, M. Basire and P. Parneix, J. Phys. Chem. A, 2011, 115, 8845-8854.

67 M. Sierka, J. Döbler, J. Sauer, G. Santambrogio, M. Brümmer, L. Wöste, E. Janssens, G. Meijer and K. R. Asmis, Angew. Chem., Int. Ed., 2007, 46, 3372.

68 G. Santambrogio, E. Janssens, S. Li, T. Siebert, G. Meijer, K. R. Asmis, J. Döbler, M. Sierka and J. Sauer, J. Am. Chem. Soc., 2008, 130, 15143. 